\title{
Physicians' medication prescribing in primary care in Riyadh city, Saudi Arabia. Literature review, part 2: rational prescribing
}

T. Khoja, ${ }^{1}$ N.A. Qureshi, ${ }^{2}$ Y. Neyaz, ${ }^{3}$ M.A. Magzoub, ${ }^{4}$ A. Haycox ${ }^{5}$ and T. Walley ${ }^{6}$

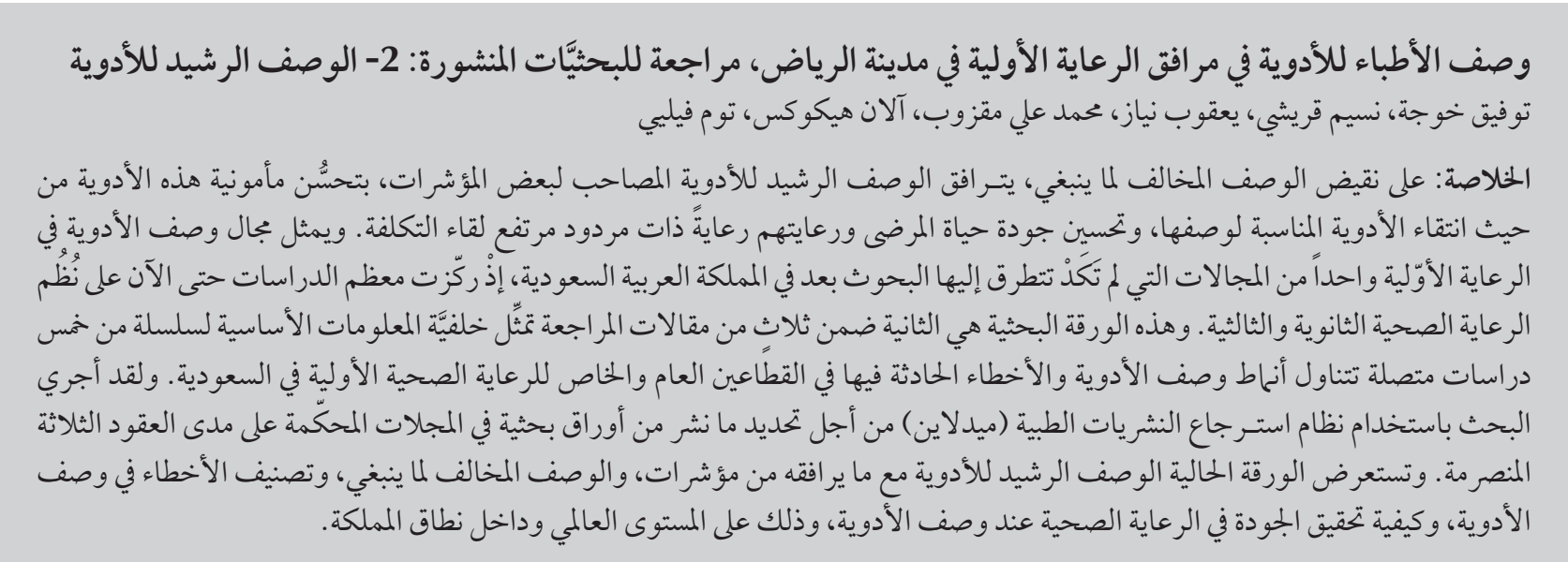

ABSTRACT Unlike sub-optimal prescribing, rational prescribing, coupled with certain indicators, is associated with improved safety in drug use in terms of selecting appropriate drug for prescribing, better quality of life for patients and cost-effective care. Medication prescribing is a relatively unexplored area of research in Saudi Arabia and until now most studies have been in the secondary and tertiary health care system. This paper is the second of 3 review articles that form the background for a series of 5 interconnected studies of prescribing patterns and medication errors in the public and private primary health care sectors of Saudi Arabia. A MEDLINE search was conducted to identify papers published in peer-reviewed journals over the previous 3 decades. The paper reviews rational prescribing with its indicators, suboptimal prescribing, classification of medication errors, and how to achieve quality in health care prescribing worldwide and in Saudi Arabia.

Comportement prescripteur des médecins en soins de santé primaires à Riyadh (Arabie saoudite). Examen des publications, deuxième partie : la prescription rationnelle

RÉSUMÉ Contrairement à la prescription non optimale, la prescription rationnelle couplée à certains indicateurs est associée à une plus grande sécurité de l'utilisation des médicaments quant au choix approprié des médicaments à prescrire, ainsi qu'à une meilleure qualité de vie des patients et un rapport coût-efficacité des soins. La prescription de médicaments est un domaine de la recherche relativement peu étudié en Arabie saoudite et, jusqu'à présent, la plupart des études ont été menées dans le système de soins de santé secondaires et tertiaires. Le présent article est le deuxième de trois articles de synthèse qui constituent le contexte de recherche d'une série de cinq études interdépendantes sur les modes de prescription et les erreurs de médication dans les secteurs public et privé des soins de santé primaires en Arabie saoudite. Une recherche sur MEDLINE a été réalisée pour identifier les travaux publiés dans des revues pratiquant l'examen collégial au cours des trente dernières années. Le présent article analyse la prescription rationnelle et ses indicateurs, la prescription non optimale, la classification des erreurs de médication, et les moyens permettant de réaliser une prescription de qualité en soins de santé dans le monde et en Arabie saoudite. 


\section{Introduction}

Rational prescribing by physicians is often described as an absence of irrational prescribing; in other words, it is easier given our current state of knowledge to define what is bad prescribing of medications rather than what is good prescribing. Bad prescribing can be defined by any number of indicators: the prescribing of an excessive range of drugs, the underuse of effective drugs, the overuse of drugs of limited clinical value, not prescribing drugs by generic name, and so on.

Most existing indicators for assessing rational prescribing focus on the cost of medications or the choice of drugs; others focus on quality of care, as noted in documents that emphasize the process of prescribing [1]. The UK Audit Commission defined rational prescribing as low-cost prescribing, while acknowledging that there may be valid reasons for higher cost prescribing at times [2]. Avery reviewed the many attempts to derive simple formulae to interpret prescribing from the UK's prescribing analysis and cost tabulation (PACT) data and concluded that existing prescribing indicators included few of the key attributes of rational prescribing and could not be used as management tools for monitoring rational prescribing $[3,4]$.

Physicians' views of what is rational prescribing tend to focus less on the choice of drug and more on the appropriateness of the drug. Parish described it as the prescription of drugs that were: appropriate, safe, effective and the least expensive for the purpose [5]. Bradley noted that there was a lack of empirical evidence on how best to judge quality in prescribing, and favoured a definition that involved balancing the evidence about the most effective way to treat a medical condition against the associated costs [6]. Barber updated the Parish definition by criticizing its focus on the prescriber and on managerial control of over-prescribing and its costs. He acknowledged that the various domains of what might be called good prescribing — maximizing effectiveness, minimizing risks, minimizing costs and respecting patient choice-could conflict with one another [7]. Good prescribing then becomes a balancing act of these domains and this model replicates the key elements of medical ethics: beneficence, nonmaleficence, distributive justice and autonomy [8].

Anotherprofessional view of rational prescribing was presented by Marinker and Reilly, who argued that it can only occur in the context of rational management of the patient, rational diagnosis etc., and therefore cannot be determined from crude data such as PACT [9]. Rational prescribing starts with a rational formulation of the patient's problem, especially a rational diagnosis, i.e. one that is defensible and can be confidently supported by evidence. This provides clarity of therapeutic intention on behalf of the prescriber. Rational prescribing also inherently has a time dimension: choosing the optimum point to initiate the therapy, the optimum duration of therapy and the optimum point to stop the medication. Rational prescribing requires not only control mechanisms that encourage compliance but also educational activities that encourage an active process of judging the appropriateness of the diagnosis and the therapy. Rational prescribing also needs to be judged on the basis of the complex interactions that take place in the consultation. PACT data alone can identify certain aspects of irrational prescribing, but are limited. Detailed medical audit on a case-by-case basis gets closest to the patient's actual complaint and the optimum treatment for it and this may be the best we can do in systems of clinical governance.

In developing countries, rational prescribing may be defined by what can realistically be measured. The World Health Organization (WHO) defined rational prescribing as "the 5 rights": the right drug, right dose, right route, right time and right patient [10]. This definition may be criticized since it assumes a clear diagnosis of the patient's problem rather than a "best formulation" of care as in the UK models of rational prescribing. Nevertheless, it is reasonable that both a clear diagnosis and a formal treatment plan should be the foundations of rational prescribing.

Compared with more industrialized countries there are few studies from Gulf countries that explore rational prescribing. This paper is the second of 3 review articles that form the background for a series of 5 interconnected studies of prescribing patterns and medication errors in the public and private primary health care sectors of Saudi Arabia [11-15].

\section{Indicators for rational prescribing}

A key definition of rational prescribing must rest on what can actually be measured. Two measures are commonly used: the medication appropriateness index (MAI), developed by Hanlon et al. [16] and the prescribing appropriateness indicators (PAI), developed by Cantrill et al. to assess long-term prescribing [1]. They are similar in content and considered by some commentators to put too much emphasis on the pharmacological aspects of prescribing [17]. The key elements are: indications; effectiveness; dosage; directions; drug interactions; drug-disease interactions; expense; practicality; duplication; and duration [18]. The UK the Audit Commission's definition of rational prescribing, which focused on costs [2], has been controversial. WHO produced a list of 12 external indicators under 3 headings - prescribing, patient care, facility [19]. While these may help in assessing prescribing by individual physicians the value of the data are hampered by their widely divergence across developed and developing countries. 


\section{Prescribing and drug choice}

A key aspect of rational prescribing is the choice of drug(s) (assuming that both doctor and patient have decided a drug is necessary). This might seem to be a simple decision using evidence-based medicine, but is in fact more complex [20]. The main factors that a physician needs to take account of when choosing a drug have been defined as: evidence of effectiveness and safety; tolerability; compliance; ease of prescription; supply; and cost [21]. These doctor-drug factors, however, are compounded by influences from patients, other doctors and professional sources, pharmaceutical companies, government and health insurers, mass media and personal experience. Physicians use negotiations with the patient, consultations with other physicians working in hospitals and primary health care and proper scrutiny of pharmaceutical information to reduce these pressures.

Physicians prescribe from a personal drugs set or formulary. They may prescribe new drugs either as a oneoff or to allow them to reshape their personal drug set. This personal set is in part formed during early medical training and then modified by all of the influences outlined above, especially by personal experience. The formulary may also be shaped by external forces, e.g. imposed by the government, as occurs in publicly-funded care in Saudi Arabia and elsewhere, or by insurance schemes in the USA. While a physician's prescribing behaviour tends to default back to the personal, he/she must always question whether the drug is right for each patient. WHO have a well-developed teaching system for medical students around the establishment of a "P (personal) drug" set and the decision about whether a drug is appropriate for an individual patient [19]. This scheme was used later in a modified form as an intervention to try to bring about changes in prescribing behaviour. In practice, doctors adopt a range of short cuts and rules of thumb to make the process of selecting a drug faster and easier. Such decision-making shortcuts represent a variety of decisionmaking strategies for prescribing which combine pragmatic, analytic, intuitive and emotional influences [20].

Decision-making in prescribing is a dynamic, complex process. Decision analysis has been defined as comprising 5 steps [22]: define the problem and the objectives; structure the decisionmaking problem; identify all possible alternatives and relevant criteria; estimate the probability that a particular option will lead to a specific outcome; evaluate the benefits of each possible outcome; and choose the best option by combining probabilities. In addition to these steps, for prescribing decisions we could add the need for monitoring (step 6), feedback (step 7) and corrective action (step 8).

\section{Achieving quality in health care}

Both the providers and consumers of health care can contribute to improving the quality of services and hence patient quality of life by setting standards. Excellent quality health services must address the needs not only of those who need the service most but also those who need it least $[23,24]$. Currently, in Saudi Arabia people with sufficient funds have access to both public and privately funded health care; those who do not have access only to publicly funded health care. Arguably health services should focus on the poor rather than the rich and any element of cost should not discriminate, since the poorer sectors of the community tend to suffer the greatest morbidity and hence have more treatment needs. Not all countries follow with this fundamental principle, e.g. in the US, where ability to pay is still the key to access to high quality services. Most publicly funded health services in
Europe, however, do subscribe to this approach, as do those in wealthier developing countries. Poorer developing countries in contrast often have to introduce user charges which limit access to care. Cost is almost always an issue in every country [25]. As a result, the best possible quality of services should be delivered within the limits and directives set by higher authorities, usually governments, although this does not exclude exceptional requests for greater funding from health professionals, health providers and health consumers.

Two complementary approaches to promote the quality of health care have been developed [26,27]. First there is the development and use of benchmarking to generate standards and targets against which the performance of health care providers can be evaluated. The second approach is to evaluate the quality of the services from the perspective of the ultimate consumer of the service: the patient. Quality assurance in health care should have a sound foundation in terms of the health system design, coupled with continuing performance assessment that should lead to appropriate educational-motivational activities and refinements in the system design, either in performance or standards or both [28]. Furthermore, quality assurance should deal with the real health problems of consumers and communities within the limits of the available resources and assess different outcomes with the goal of satisfying patients $[26,29,30]$ and evaluating patient care safety [31].

\section{Achieving quality in prescribing \\ Educational and information approaches}

One approach to improving quality in practice is educating doctors to change their behaviour. A Cochrane review of the effects of continuing education on professional practice and health care 
outcomes concluded that interactive workshops could result in moderately large changes in professional practice, whereas didactic sessions alone were unlikely to be effective [32].

A study by Allery et al. involving 50 general practitioners (GPs) and 50 consultants sought to describe the complete range of factors that doctors recognize as changing their clinical practice and to provide a measure of how often education is involved in change [33]. The 3 most frequently mentioned reasons for changes in clinical practice were: organizational factors; education; and contact with professionals. Consultants stated that they were influenced by medical journals and scientific conferences, while GPs thought they were more influenced by medical newspapers and postgraduate meetings. The authors concluded that education is involved in about a third of changes in clinical practice and that the wide range of other factors affecting changes in practice need to be taken into account in providing and evaluating education.

In another study a qualitative interview survey was made of 18 GPs to determine why they had recently changed their prescribing practices [34]. The researchers concluded that many factors are involved, and indentified 3 main models of change - the accumulation model, the challenge model and the continuity model-noting too that positive feedback reinforces prescribing practice.

Cantillon and Jones's review of the literature summarized the effectiveness of continuing medical education (CME) in the context of UK general practice [35]. This review indicated that the most effective methods for bringing about change in GPs' behaviour were: learning linked to clinical practice; interactive educational meetings; outreach events; and strategies that involve multiple educational interventions. The authors noted that the least effective methods for CME were those most commonly used in general practice.
Soumerai and Lipton critically reviewed the available evidence on drug information bulletins, guidelines and other professionally prepared materials. They observed that printed materials on their own generally have limited, or no, effectiveness in influencing changes in prescribing behaviour and that any noticeable effect was usually short-term [36]; similar conclusions were reached in a Cochrane review [37].

Educational outreach visits, however, can have an effect on prescribing behaviour, particularly when combined with social marketing related to a socially beneficial change [32]. This is the basis of the system of visits by prescribing advisers in the UK [8]. However, educational outreach was associated with only a $7 \%$ increase in the desired professional practice $[38,39]$.

\section{Administrative approaches}

Administrative approaches can also be used to improve quality in practice [40]. Examples include incentive schemes such as the GP fund-holding scheme in the UK $[41,42]$, in which changes in prescribing practice, albeit limited in magnitude and sustainability, were achieved by simple measures, e.g.introducing generic prescribing [41]. The opposite of providing incentives is to provide disincentives, as attempted in France and in Germany. These have achieved mixed results and engendered considerable dissatisfaction from doctors.

Another aspect to the administrative approach is providing feedback to doctors on their prescribing habits. Harris et al. described this as a powerful but transient approach to changing prescribing practice that can only succeed if there are professional incentives $[43,44]$. The feedback approach can be useful as part of a multifaceted approach to achieving changes in professional practice [45].

Even if prescribing can be improved by educational methods and to a lesser extent by managerial actions, these approaches are more likely to be influential on what drugs are prescribed rather than on whether a drug needs to be prescribed at all. Formularies are a good example of this [8]. Clinical guidelines may also be effective and might bring about greater changes, but only if they are acceptable to the profession [38]. In future, computerized decision support systems may be the most useful tool [8], but these are unlikely to be implemented in Saudi Arabia in the near future. Better quality prescribing in Saudi Arabia may be as simple as encouraging proper documentation of prescriptions.

\section{Recommendations for optimizing prescribing and patient safety}

The need to improve patient safety by reducing prescribing errors and adverse drugs events (ADEs) has been increasingly recognized by the medical community. This concern was highlighted after the publication of recommendations from the Institute of Medicine [46] which were supported by other investigators $[47,48]$. These focused on enhancing the knowledge base about safety; identifying and learning from errors; raising standards and expectations for improvements in safety; and creating safety systems inside health care organizations. Besides the domains of patient safety and organization assessment tools, the Institute rightly identified the roles of the public, the media and stakeholders from different disciplines.

Grube has commented about the deficiencies in the reporting of prescribing errors and identified barriers to reporting errors - e.g. physician's fears of legal vulnerability, the culture of health care providers and an organization's unwillingness to take corrective actiontogether with possible solutions [47]. In the UK, the National Patient Safety Agency has been set up to address system errors in drug prescribing and medication use, and a target was set to 
reduce the number of serious errors by $40 \%$ by the year 2005 . This may be difficult in the UK where the rate of medication errors was reported in one hospital to be $3.0 \%$, significantly lower than the rate of $6.9 \%$ reported in a US hospital with a typical unit dose drug distribution system [49].

\section{Categorizing suboptimal prescribing}

In any analysis of suboptimal prescribing, it is crucial to distinguish clearly between prescribing errors, dispensing errors and ADEs. Notably, accurate definition of the prescribing problems being addressed is crucial to the development of effective solutions.

A number of terms are used in defining suboptimal prescribing. The term "patient safety incident" is an umbrella term to describe a single incident or a series of incidents that occur over time $[50]$ and is preferred to the terms "adverse event", "clinical error" or "near miss", as such terms suggest individual causality and blame. The concept of "trigger data" has been developed to reflect the use of clinical data which indicates the probability of an $\mathrm{ADE}$ developing. For example, a medication, laboratory value or other indicator that appears to be exceptional would prompt a further review of patient care.

ADEs rarely have single causes and the doctrine of causation is used to relate a range of factors including predisposing, enabling, precipitating or reinforcing factors to the occurrence of such events [51]. It is important, therefore, to investigate a wide range of factors in suboptimal prescribing. Each contributing factor represents an active failure, such as a situational factor or a latent condition that played a role in the suboptimal outcome [51]. In addressing suboptimal prescribing, it is essential that a culture of safety is encouraged throughout the whole organization.
This requires the development of an integrated pattern of individual and organizational behaviour that continuously seeks to minimize any form of patient harm resulting from care [52]. "Dispensing errors" are a deviation from an interpretable written prescription or medication order, including written modification of the prescription made by a pharmacist following contact with the prescriber or in compliance with the pharmacy policy. Any deviation from professional or regulatory references, or guidelines affecting dispensing procedures, would also be defined as representing a dispensing error [53].

A "drug-related problem" is defined as an event or circumstance that actually or potentially interferes with the desired health outcomes arising from drug therapy [54] and adversely affects the benefit-risk ratio for an individual patient. "Prescribing errors" take many forms: a drug at the wrong time, administering it in the wrong dosage or administering it by the wrong route. An error of omission occurs when an ADE results from an action not taken; for example, when a dose of a medication should be administered but is omitted [55]. Failure mode and effects analysis (FMEA) is a risk assessment method based on the simultaneous analysis of failures modes, their consequences and their associated factors and is used to attempt to identify and prevent problems before they occur. A wide range of alternative risk assessment methods using the failure mode technique exist, all of which assume that prescribing errors are inevitable and that the aim must be to identify systems by which such errors can be minimized. A "just" culture is a key element of a "safe" culture that reconciles professional accountability within a system which balances the need to learn from mistakes and the need for disciplinary action [56]. A just culture hinges on a collectively agreed and clearly understood distinction being drawn between acceptable and unacceptable behaviour [57]. Marx has expanded the concept further and provided practical guidance on its use in health care organizations [56]. "Error conditions" are defined as errors in design, organization, training or maintenance that may lie dormant in systems for long periods of time [46]. Unlike active failures, latent conditions can be identified and remedied before an $\mathrm{ADE}$ occurs, enabling proactive rather than reactive risk management [58]. Latent errors have been described as "accidents waiting to happen" [59] and can translate into error-provoking conditions within the local workplace or long-lasting weaknesses in the defences of the system.

A "medication error" can be defined as any preventable event that leads to inappropriate medication use while the medication is in the control of the health care provider or consumer. The medication use system represents a combination of interdependent processes: selecting and procuring; storage; prescribing; transcribing and verifying; preparingand dispensing; administering and monitoring [60]. Each major process presents its own unique opportunities for error [61]. A "monitoring error" occurs as a result of failure to review a prescribed regimen for appropriateness and for detecting problems, or failure to use the appropriate clinical or laboratory data for adequate assessment of the patient response to the prescribed therapy. An "opportunity for error" is the basic unit of data in medication error studies and is defined as any dose or doses given, plus any dose ordered but omitted $[62,63]$.

\section{Taxonomy of medication errors}

Not all ADEs are due to prescribing errors. There are many types of clinical errors with multiple etiologies. There are also several taxonomies of medication errors and a number of authors have 
described different ways of classifying clinical and prescribing errors.

- Clinical importance of the event. Neville et al. classified clinical errors into 4 major groups: type A are potentially serious to patients; type $B$ are a major nuisance that require contact between the pharmacist and prescribing doctor for clarification; type $\mathrm{C}$ are a minor nuisance where the pharmacist must use his/her professional judgement when dispensing; and type $\mathrm{D}$ are trivial [64].

- Dispensing errors. Ross etal. in a study of a paediatric hospital have classified medication errors, largely dispensing errors, into 8 types: wrong medication; wrong dose of medication; wrong concentration of medication; wrong fluid or wrong rate of injecting medication; medication administered at a very different time from when it was prescribed; medication likely to produce a known allergic reaction; medication given but not prescribed or vice versa; wrongly labeled and wrong medicine or strength of medicine dispensed for use at home. [65].

- Effectiveness and safety or cost-effectiveness. Soumerai and Lipton highlighted common prescribing errors in terms of: use of toxic or addictive drugs when safer agents are available; use of drug therapy when no therapy is required; use of an ineffective drug; use of a costly drug when a less expensive preparation would be just as effective; suboptimal or excessive use of effective agents; failure to discontinue therapy when the drug is no longer needed; and failure to introduce new and effective drugs into practice. This might be extended to include any under-use of an effective drug [36].

- Health care quality problems. The IOM developed the approach of Soumerai and Lipton [36] and described 3 types of health care quality problems and prescribing errors - under-use, overuse, misuse - all of which need to be addressed to improve patient safety $[66,67]$. Reported misuse is closer to what Neville et al. and Ross et al. recognize in terms of actually making an error, e.g. prescribing the wrong drug or dose $[64,65]$.

- Pharmacy errors. Kelly, after reviewing 17 studies discussed the contribution of the hospital pharmacy to preventing or causing $\mathrm{ADEs}$ and classified pharmacy errors into the following: errors due to system failure; errors due to insufficient supervision or staffing; and errors by pharmacy personnel [68].

- Allergies or interactions. Rozich and Resar documented several types of medication errors, including allergies or interactions: errors related to drug, dose, drug administration; medication administration records; allergy or interactions; and others [69].

- Psychological. Other researchershave given a psychological classification of medication errors, drawn not from the prescribing literature but more broadly from the literature of industrial safety [70]. Such mistakes include knowledge-based and role-based errors: mistakenly applying a good rule, e.g. injecting anti-inflammatory drug diclofenac into the lateral thigh (the usually preferred site) rather than the buttock (which is recommended for diclofenac); or applying a bad rule, e.g. when excessive doses of the ACE inhibitor captopril were used when it was first introduced.

- Underlying cause. Dovey et al. undertook a detailed analysis of medical errors in family practice [71]. Errors were categorized in relation to their underlying cause, primarily as due to problems with processes involved in carrying out general practice and skills involved in general practice.

Although the adopted taxonomy of prescribing errors certainly differ across studies, all of them to some extent help in identifying the causes of errors and methods to reduce them. Both prescribing errors and ADEs are preventable and need proper investigation by the relevant authorities using standardized protocols.

In summary, rational medication prescribing is closely linked with patient safety and quality of life. Suboptimal prescribing, especially medication errors, are classified variously by different researchers. The common theme, however, is the need to identify the causes out of the range of possibilities and to create practical systems for preventing them.

\section{References}

1. Cantrill J, Sibbald B, Buetow S. Indicators of the appropriateness of long term prescribing in general practice in the United Kingdom: consensus development, face and content validity, feasibility and reliability. Quality in Health Care, 1998, 7:130-135.

2. Audit Commission. A prescription for improvement: towards more rational prescribing in general practice. London, HMSO, 1994.

3. Avery AJ et al. Investigating relationships between a range of potential indicators of general practice prescribing: an observational study. Journal of Clinical Pharmacy and Therapeutics, 1998a, 23:441-450.

4. Avery AJ. Appropriate prescribing in general practice: Development of the indicators. Quality in Health Care, 1998b, 7:123.
5. Parish P. Drug prescribing: the concern of all. Royal Society of Health Journal, 1973, 493:213-217.

6. Bradley CP. Decision making and prescribing patterns-a literature review. Family Practice, 1991, 8:276-287.

7. Barber N. What constitutes good prescribing? British Medical Journal, 1995, 310:923-925.

8. Chapman S. Educational outreach in medicines management. In: Panton R, Chapman S, eds. Medicines management. London, British Medical Journal Books, 1998.

9. Marinker M, Reilly P. Rational prescribing: How can it be judged? Controversies in health care policies: challenges to practice. London, British Medical Journal Publishing Group, 1994:89-110. 
10. Benjamin DM. Reducing medication errors and increasing patient safety: case studies in clinical pharmacology. Journal of Clinical Pharmacology, 2003, 43:768-783.

11. Neyaz $\mathrm{Y}$ et al. Medication prescribing pattern in primary care in Riyadh city, Saudi Arabia. Eastern Mediterranean Health Journal, 2011, 17(2):149-155.

12. Khoja T et al. Medication errors in primary care in Riyadh city, Saudi Arabia. Eastern Mediterranean Health Journal, 2011, 17(2):156-159.

13. Magzoub MA et al. Determinants of physicians' medication prescribing behaviour in primary care in Riyadh city, Saudi Arabia. Eastern Mediterranean Health Journal, 2011, 17(2)160-166.

14. Neyaz Y et al. Predictors of quality of medication prescribing in primary care in Riyadh city, Saudi Arabia. Eastern Mediterranean Health Journal, 2011, 17(2):67-171.

15. Qureshi NA et al. Effectiveness of three interventions on primary care physicians' medication prescribing in Riyadh city, Saudi Arabia. Eastern Mediterranean Health Journal, 2011, 17(2):172-179.

16. Hanlon JT et al. Method for assessing drug therapy appropriateness. Journal of Clinical Epidemiology, 1992, 45:1045-1051.

17. Barber $\mathrm{N}$ et al. Measuring the appropriateness of prescribing in primary care: are current measures complete? Journal of Clinical Pharmacy and Therapeutics, 2005, 30:533-539.

18. Chapman S, Durieux P, Walley T. Good prescribing practice. In: Regulating pharmaceuticals in Europe: striving for efficiency, equity and quality, Mossialos E, Mrazek M, Walley T, eds. MiltonKeybes, UK, Open University Press, 2004.

19. How to investigate drug use in health facilities: selected drug use indicators. Geneva, World Health Organization, 1993 (WHO/ DAP/93.1).

20. Denig P, Bradley C. How doctors choose drugs. In: Hobbs $\mathrm{R}$, Bradley C, eds. Prescribing in primary care. Oxford, Oxford University Press, 1998.

21. Patrick RW. Medication errors: protecting both patient and provider. Emergency Medical Services, 2003, 32:40-42.

22. Abelson RP, Levi A. Decision making and decision theory. In: Lindzey G, Aronson E. The Handbook of Social Psychology. New York, Random House, 1995.

23. Ovretveit J. Health service quality: an introduction to quality methods for health sciences. London, Blackwell Science, 1992.

24. Roemer MI, Montoya-Aguilar C. Quality assessment and assurance in primary health care. Geneva, World Health Organization, 1988.

25. Maynard A, Bloor K, Freemantle N. Challenges for the National Institute for Clinical Excellence. British Medical Journal, 2004, 329:227-229.

26. Report of the WHO working group on quality assurance. Geneva, World Health Organization, 1994:18-20.

27. Davies HTO, Crombie IK. Assessing the quality of care: measuring well supported process may be more enlightening than monitoring outcomes. British Medical Journal, 1995, 311:766.

28. Ellis R, Whittington D. Quality assurance in health care. In: $A$ handbook of quality assurance. London, Edward Arnold, 1993.

29. Scott A, Smith RD. Keeping the customer satisfied: issues in the interpretation and use of patient satisfaction surveys. International Journal for Quality in Health Care, 1994, 6:353-359.

30. Donabedian A. Quality assurance in health care: consumers role. Quality in Health Care, 1992, 1:247-251.

31. Effken JA et al. Using OrgAhead, a computational modelling program, to improve patient care unit safety and quality Outcome. International Journal of Medical Informatics, 2005 74:605-613.
32. O'Brien MA et al. Continuing education meetings and workshops: effects on professional practice and health care outcomes. Cochrane Database of Systematic Reviews, 2001, (2):CD003030.

33. Allery LA, Owen PA, Robling MR. Why general practitioners and consultants change their clinical practice: a critical incident study. British Medical Journal, 1997, 314:870-874.

34. Armstrong D, Reyburn H, Jones R. A study of general practitioners' reasons for changing their prescribing. British Medical Journal, 1996, 312:949-952.

35. Cantillon $\mathrm{P}$, Jones $\mathrm{R}$. Does continuing medical education in general practice make a difference? British Medical Journal, 1999, 318:1276-1279.

36. Soumerai SB, Lipton HL. Evaluating and improving physician prescribing. In: Strom BL, ed. Pharmacoepidemiology, 2nd ed. London, John Wiley, 1994.

37. Farmer $\mathrm{N}$ et al. Printed educational materials: effects on professional practice and health care outcomes. Cochrane Database of Systematic Reviews, 2008, 3: CD004398.

38. Freemantle $\mathrm{N}$ et al. A randomised controlled trial of the effect of educational outreach by community pharmacists on prescribing in UK general practice. British Journal of General Practice, 2002, 52:290-295.

39. Bero LA et al. Getting research findings into practice: Closing the gap between research and practice: an overview of systematic reviews of interventions to promote the implementation of research findings. British Medical Journal, 1998, 317:465-468.

40. Chaix-Couturier $\mathrm{C}$ et al. Effects of financial incentives on medical practice: results from a systematic review of the literature and methodological issues. International Journal for Quality in Health Care, 2000, 12:133-142.

41. Wilson R, Walley T. Prescribing costs in general practice fundholding. Lancet, 1995, 346:1710-1711.

42. Harris CM, Scrivener G. Fundholders' prescribing costs: the first five years. British Medical Journal, 1996, 313:1531-1534.

43. Harris $\mathrm{CM}$ et al. Prescribing-a suitable case for treatment. Journal of the Royal College of General Practitioners, 1984, 24:1-39.

44. Harris CM et al. Prescribing-a case for prolonged treatment. Journal of the Royal College of General Practitioners, 1985, 35:284-287.

45. Jamtvedt $\mathrm{G}$ et al. Audit and feedback: effects on professional practice and health care outcomes Cochrane Database of Systematic Reviews, 2003, 3:CD000259.

46. Kohn LT, Corrigan JM, Donaldson MS, eds. To err is humanbuilding a safer health system. Washington DC, National Academy Press, 2000:23.

47. Grube JA. Learning from healthcare errors: effective-reporting systems. Journal for Healthcare Quality, 2001, 23:25-29.

48. Wilson NJ, Hatlie MJ. Advancing patient safety: a framework for accountability and practical actions. Journal for Healthcare Quality, 2001, 23:30-34.

49. Dean BS et al. Comparison of medication errors in an American and a British Hospital. American Journal of Health-System Pharmacy, 1995, 52:2543-2549.

50. Seven steps to patient safety: full reference guide. London, National Patient Safety Agency, 2004.

51. Wade J, ed. Building a safer system. A national integrated strategy for improving patient safety in Canadian health care. Ottawa, National Steering Committee on Patient Safety. 2002.

52. Aspden P et al., eds. Patient safety: achieving a new standard for care. Washington DC, National Academy Press, 2004.

53. Beso A, Franklin BD, Barber N. The frequency and potential causes of dispensing errors in a hospital pharmacy. Pharmacy World and Science, 2005, 27:182-190. 
54. PNCE classification for drug-related problems, V5.00. Zuidlaren, The Netherlands, Pharmaceutical Care Network Europe, 2003.

55. Sentinel events policy and procedures. Oakbrook Terrace, IIlinois, Joint Commission on Accreditation of Healthcare Organizations, 2002.

56. Marx D. Patient safety and the "just culture": a primer for health care executives. New York, Columbia University, 2001:28.

57. Reason JT, Hobbs A, eds. Managing maintenance error. Aldershot, UK, Ashgate, 2003:148.

58. Reason JT. Human error: models and management. British Medical Journal, 2000, 320:768-770.

59. Leape LL. Preventing adverse drug events. American Journal of Health-System Pharmacy, 1995, 52:380.

60. Cohen MR, ed. Medication errors. Washington DC, American Pharmaceutical Association, 1999.

61. Leape LL et al. Reducing adverse drug events. Breakthrough series guide. Boston, Institute for Healthcare Improvement, 1998:84-91.

62. Allan EL, Barker KN. Fundamentals of medication error research. American Journal of Hospital Pharmacy, 1990, 47:555-571.

63. Barker KN, Kimbrough WW, Heller WM. The medication error problem in hospitals: I The detection and the use of errors a measurement of medication systems. Hospital Formulary, 1966, 1:29-34.

64. Neville RG et al. A classification of prescription errors. Journal of the Royal College of General Practitioners, 1989, 39:110-112.

65. Ross LM, Wallace J, Paton JY. Medication errors in a Paediatric Teaching hospital in the UK: five years operational experience. Archives of Disease in Childhood, 2000, 83:492-497.

66. Chassin MR, Galvin RW. The urgent need to improve health care quality. Institute of Medicine national round-table on health care quality. Journal of the American Medical Association, 1998, 280:1000-1005.

67. Little $\mathrm{P}$ et al. Open randomized trial of prescribing strategies in managing sore throat. British Medical Journal, 1997, 314:722.

68. Kelly WN. Pharmacy contributions to adverse medication events. American Journal of Health-System Pharmacy, 1995, 52:385-390.

69. Rozich JD, Resar RK. Medication safety: one organization's approach to the challenge. Journal of Clinical Outcomes Management, 2001, 8:27-34.

70. Ferner RE, Aronson JK. Medication errors, worse than a crime. Lancet, 2000, 355:947-948.

71. Dovey SM et al. A preliminary taxonomy of medical errors in family practice. Quality and Safety in Health Care, 2002, $11: 233-238$

\section{The International Pharmacopoeia}

The International Pharmacopoeia (Ph. Int.) comprises a collection of quality specifications for pharmaceutical substances (active ingredients and excipients) and dosage forms together with supporting general methods of analysis, that is intended to serve as source material for reference or adaptation by any WHO Member State wishing to establish pharmaceutical requirements.

The activities related to The International Pharmacopoeia are an essential element in the overall quality control and assurance of pharmaceuticals contributing to the safety and efficacy of medicines. The selection of monographs for inclusion in The International Pharmacopoeia recognizes the needs of specific disease programmes and the essential medicines nominated under these programmes; it is based primarily on those substances included in the current WHO Model List of Essential Medicines.

The Pharmacopoeia and related documents can be downloaded from: http://www.who.int/medicines/publications/pharmacopoeia/overview/en/index.html 